\title{
Pericarditis purulenta en la infancia Análisis clínico
}

DRTS. V. PACHECO *, S. CERESA*, G. VENEGAS *, H. SUAREZ *,P. ARAYA ** y A. URRIZOLA**.

La pericarditis purulenta es una afección que puede presentarse a cualquier edad, siendo más frecuente en los primeros años de vida.

Suele ser causada por organismos Gram $(t)$ como estafilococos, neumococos y estreptococos; los bacterios Gram (-), incluyendo hemophilus influenzae, E. Coli, meningococos y salmonellas son de frecuencia más rara, aunque también pueden intervenir. El microorganismo puede alcanzar el pericardio, por una propagación directa desde una neumonía, habitualmente con empiema o en el curso de una septicemia aguda.

El propósito de nuestro estudio es presentar los resultados de una experiencia de 10 años en la edad pediátrica destacando los hechos fundamentales para su diagnóstico clínico oportuno y revisar la terapia empleada,

\section{MATERIAL Y METODO:}

Se analizan 11 casos hospitalizados en la Unidad de Infecciosos del Servicio de Pediatría del Hospital Guillermo Grant B. de Concepción en los últimos 10 años (1965-1974).

El diagnóstico de pericarditis se basó en elementos clínicos, radiológicos, electrocardiográficos y de procedimientos como pericardiocentesis. Se establece la puerta de entrada y la bacteriología causante de la afección.

\section{RESULTADOS:}

La distribución por edad, sexo y estado nutritivo se aprecia en la tabla 1.

\footnotetext{
*: Becario Universidad de Concepción. Departamento de Pe-

**: Servicio de Pediatria. Hospital Guillermo Grant Benavente,
Concepción. Concepción.
}

\section{TABLA 1}

Distribución por edad, sexo y estado nutritivo de 11 niños con pericarditis purulenta

\begin{tabular}{|c|c|c|c|c|c|}
\hline \multirow{2}{*}{$\begin{array}{c}\text { EDAD } \\
\text { años }\end{array}$} & \multirow[b]{2}{*}{ casos } & \multicolumn{2}{|c|}{ SEXO } & \multirow{2}{*}{$\begin{array}{l}\text { ESTADO } \\
\text { Satisfact. }\end{array}$} & \multirow{2}{*}{$\begin{array}{l}\text { NUTRIT. } \\
\text { Deficiente }\end{array}$} \\
\hline & & $\mathbf{F}$ & $\mathbf{M}$ & & \\
\hline $0 \cdot 2 \mathrm{a}$ & 1 & 1 & 0 & 0 & $1 \mathrm{D} \mathrm{III}$ \\
\hline 2 a $1 m-4$ & 3 & 1 & 2 & 1 & $\begin{array}{l}2 \text { sub- } \\
\text { alim. }\end{array}$ \\
\hline 4 a $1 m-6$ & 2 & 0 & 2 & 2 & 0 \\
\hline 6 a $1 \mathrm{~m}-8$ & 4 & 3 & 1 & 4 & 0 \\
\hline 8 a $1 \mathrm{~m}-10 \mathrm{a}$ & 0 & 0 & 0 & 0 & 0 \\
\hline 10 a $1 \mathrm{~m}-12 \mathrm{a}$ & 1 & 0 & 1 & 1 & 0 \\
\hline TOTAL & 11 & 5 & 6 & 8 & 3 \\
\hline
\end{tabular}

Las edades fluctuaron entre 1 año 4 meses y 11 años; seis de nuestros pacientes pertenecen al sexo masculino y cinco al femenino. El estado nutritivo fue calificado como satisfactorio en 8 niños $(73 \%)$; el resto se trataba de un desnutrido Grado III y dos niños presentaban una desnutrición moderada.

La procedencia de nuestros pacientes se establece en la Tabla 2.

Nueve de ellos procedían de un medio urbano y dos de rural. Sólo 2 niños eran de la ciudad de Concepción y el resto habían sido derivados de establecimientos de la región.

La pericarditis purulenta ocurrió conjuntamente durante el curso de un cuadro de septicopiohemia en diez casos y concomitante con un empiema pleural derecho en un caso.

La puerta de entrada de la septicopiohemia se pudo pesquisar en seis casos como se aprecia en la Tabla 3. Cinco provenían de un proceso cutáneo y sólo uno de una etmoiditis. 
TABLA 2

Distribución de la procedencia de 11 niños con pericarditis purulenta

\begin{tabular}{|c|c|c|c|}
\hline Procedencia & No casos & $\begin{array}{l}\text { URBANO } \\
\text { № casos }\end{array}$ & $\begin{array}{l}\text { RURAL } \\
N^{\circ} \text { casos }\end{array}$ \\
\hline Concepción & 2 & 2 & 0 \\
\hline Talcahuano & 1 & 1 & 0 \\
\hline Chiguayante & 1 & 1 & 0 \\
\hline Tomé & 2 & 2 & 0 \\
\hline Los Angeles & 1 & 1 & 0 \\
\hline Coronel & 1 & 1. & 0 \\
\hline Lota Alto & 2 & 1 & 1 \\
\hline Colcura & 1 & 0 & 1 \\
\hline TOTAL & 11 & 9 & 2 \\
\hline
\end{tabular}

TABLA 3

Distribución de la puerta de entrada en 11 niños con pericarditis purulenta

\begin{tabular}{lc}
\hline \multicolumn{1}{c}{$\begin{array}{c}\text { Con sépticopiohemia } \\
\text { puerta de entrada }\end{array}$} & Frecuencia \\
\hline Piodermitis & 3 \\
Herida infectada & 2 \\
(contusa de la rodilla, punzante del pie) & 1 \\
\hline
\end{tabular}

Sin sépticopiohemia

Pleuroneumonía y empiema derecho

1

Otras manifestaciones acompañantes de la septicopiohemia se aprecian en la tabla 4. Osteomielitis en 8 casos, neumopatía aguda en $7 \mathrm{y}$ meningitis purulenta en dos.
TABLA 4

Distribución de afecciones concomitantes en 11 niños con pericarditis purulenta

Con sépticopiohemia

Afección concomitante

Frecuencia

\begin{tabular}{|c|c|}
\hline Osteomielitis & 8 \\
\hline Neumopatía - derecha & 1) \\
\hline aguda $\quad-$ izquierda & 17 \\
\hline — bilateral & 5 \\
\hline Meningitis & 2 \\
\hline
\end{tabular}

Sin sépticopiohemia

Pleuroneumonía y empiema derecho

1

TABLA 5

Distribución del estudio bacteriológico de 11 niños con pericarditis purulenta

\begin{tabular}{lcc}
\hline Estudio bacteriológico & N: casos & $\%$ \\
\hline Positivo a estafilococo dorado & 7 & $64 \%$ \\
Negativo & 4 & $36 \%$ \\
\hline TOTAL & 11 & $100 \%$ \\
\hline
\end{tabular}

El estudio bacteriológico realizado en los 11 niños fue positivo a estafilococo dorado en $7 \mathrm{ca}$ sos $(64 \%)$ y negativos en 4 casos $(36 \%)$ como queda demostrado en la Tabla 5.

El estafilococo aureus fue aislado en el hemocultivo en 4 niños; en el pus pericárdico en 3 ; en el LCR en 2; en el pus subperióstico en 2 y en el pus pleural en uno (Tabla 6 ).

TABLA 6

Distribución de estudios bacteriológicos positivos a estafilococo dorado en 7 niños con pericarditis

purulenta

\begin{tabular}{|c|c|c|c|c|c|c|}
\hline$N:$ niños & $\begin{array}{l}\text { Hemocul- } \\
\text { tivo }\end{array}$ & $\begin{array}{c}\text { Pus } \\
\text { pericár- } \\
\text { dico }\end{array}$ & LCR & $\begin{array}{c}\text { Pus } \\
\text { osteomie- } \\
\text { litis }\end{array}$ & $\begin{array}{c}\text { Pus } \\
\text { pleural }\end{array}$ & $\begin{array}{c}\text { Total } \\
\text { cultivos }\end{array}$ \\
\hline $1^{\circ}$ & $\bullet$ & & & & & 1 \\
\hline $2^{\circ}$ & & $\bullet$ & & & & 1 \\
\hline 3 & $\bullet$ & & $\bullet$ & & & 2 \\
\hline $4 !$ & $\bullet$ & $\bullet$ & & $\bullet$ & & 3 \\
\hline $5 ?$ & $\bullet$ & $\bullet$ & $\bullet$ & & & 3 \\
\hline $6:$ & & & & $\bullet$ & & 1 \\
\hline $7{ }^{\circ}$ & & & & & - & 1 \\
\hline TOTAL: & 4 & 3 & 2 & 2 & 1 & 12 \\
\hline
\end{tabular}


TABLA 7

Distribución del antibiograma en 7 pacientes con estudio bacteriológico

\begin{tabular}{lccc}
\hline \multicolumn{1}{c}{ Antibiótico } & No casos & $\begin{array}{c}\text { Sensible } \\
\text { casos }\end{array}$ & $\begin{array}{c}\text { Resistente } \\
\text { casos }\end{array}$ \\
\hline Cloxacilina & 7 & 7 & \\
Dicloxacilina & 4 & 4 & \\
Metacilina & 4 & 4 & 7 \\
Penicilina & 7 & & \\
Eritromicina & 3 & 3 & \\
Estreptomicina & 3 & 3 & 1 \\
Tetraciclina & 4 & 4 & \\
Cloramfenicol & 5 & 4 & \\
Lincomicina & 3 & 3 & \\
Kanamicina & 4 & 4 & \\
Gentamicina & 2 & 2 & \\
\hline
\end{tabular}

En 4 niños fue aislado en una sola de estas localizaciones y en tres en varias de ellas.

E1 antibiograma practicado en los 7 pacientes demostró sensibilidad en todos ellos a Cloxacilina y resistencia también en todos a Penicilina (tabla 7).

Los síntomas y signos que presentaron los 11 pacientes con pericarditis supurada se demuestran en la Figura N 1. El síntoma fiebre se presentó en el $100 \%$ de los pacientes y la duración fue variable dependiendo de la gravedad del cuadro séptico. En todo caso más de 20 días en todos los niños. Con respecto, a la signología cadíaca, frote pericárdico se encontró en 10 de nuestros pacientes; este junto a tonos apagados, aumento del área de matidez cardíaca y desaparición del choque de la punta constituyeron los elementos fundamentales en el diagnóstico clínico de pericarditis. En 5 pacientes se hizo el diagnóstico de taponamiento cardíaco, en tres de ellos se consignó al ingreso y en el resto durante su evolución. Es interesante destacar que 7 de nuestros pacientes presentaron dolor abdominal previo al diagnóstico de pericarditis con una fluctuación de uno a cuatro días. Elementos cutáneos se

Disialoucion of los sintomas y stonos en 10 pacientes con pericanoitis pUnULENTA. DE waYOR a memok.

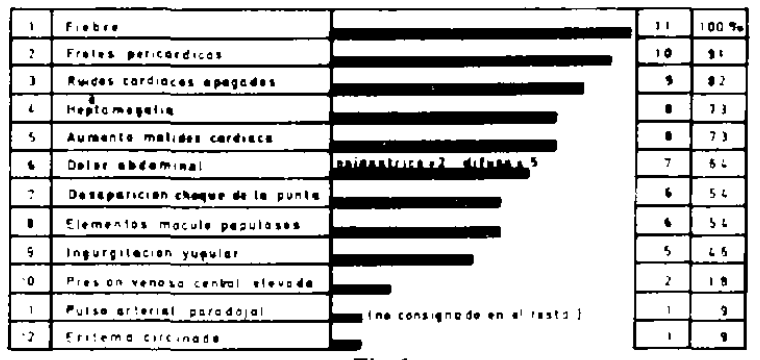

Fig 1. apreciaron 7 casos atribuibles a la etiología bacteriana del cuadro.

Los exámenes de laboratorio y procedimientos diagnósticos realizados al ingreso de los pacientes se consigna en la Tabla 8 . Se practicó estudio radiológico en 8 niños, observando aumento del tamaño de la imagen cardíaca en 7 , disminución, o ausencia de latidos en 4 y corazón de tamaño normal en uno.

El ECG se realizó en siete pacientes, los signos fueron elevación del segmento ST y onda T aplanada $\mathrm{y} / \mathrm{o}$ invertida en todos los niños, además de bloqueo de I grado en un caso.

\section{TABLA 8}

Exámenes de laboratorio realizados al ingreso con fines diagnósticos en 8 niños con pericarditis purulenta

\begin{tabular}{ll}
\hline Estudio radiológico & 8 \\
\hline Aumento de tamaño de imagen cardíaca & 7 \\
Disminución o ausencia de latidos & 4 \\
Tamaño normal del corazón & 1 \\
\hline Electrocardiograma & 7 \\
\hline Elevación segmento S T & 7 \\
Onda T aplanada y/o invertida & 7 \\
Bloqueo A-V 1 grado & 1 \\
\hline Pericardiocentesis & 2 \\
\hline Positiva & 2 \\
Negativa & 0 \\
\hline
\end{tabular}

TABLA 9

Distribución de los días de evolución del cuadro séptico previo al diagnóstico clínico de pericarditis purulenta en 10 niños

\begin{tabular}{ccc}
\hline No días & N: niños & $\%$ \\
\hline $1-3$ & 1 & 10 \\
$4-7$ & 1 & 10 \\
$+7-(11)$ & 8 & 80 \\
\hline TOTAL & 10 & $100 \%$ \\
\hline
\end{tabular}

Los días de evolución de cuadro séptico antes del comienzo de la pericarditis fueron más de 7 días en el $80 \%$ de los niños (Tabla 9).

Los días de hospitalización previos al diagnóstico de pericarditis se establecen en la Tabla 10; es de notar que en el $54 \%$ de los pacientes el diagnóstico se hizo en las primeras 72 horas de hospitalización. 


\section{TABLA 10}

Distribución de los días de hospitalización previos al diagnóstico clínico de pericarditis purulenta en 11 niños

\begin{tabular}{ccl}
\hline No días & N: niños & $\%$ \\
\hline $1-3$ & 6 & 54,5 \\
$4-7$ & 2 & 18 \\
+7 & 3 & 27,5 \\
\hline TOTAL & 11 & $100 \%$ \\
\hline
\end{tabular}

Las medidas de tratamiento fueron antibióticos, pericardiocentesis, pericardiostomía y pericardioectomía. Se empleó terapia antibiótica en todos los casos. Los antibióticos usados se expresan en la tabla 11. Diez pacientes recibieron Cloxacilina con una duración que fluctuó entre 38 y 104 días. La duración de la terapia fue variable dependiendo de la gravedad del cuadro séptico.

Pericardiocentesis se efectuó en los primeros días de hospitalización en dos pacientes (ambos con signos de taponamiento cardíaco), en uno de ellos en cinco oportunidades y en el otro por una sola vez. Ambos niños fueron sometidos a pericardiectomía posterior.

Cuatro pacientes fueron sometidos a tratamiento quirúrgico: pericardiostomía se hizo en dos, en uno de ellos al día siguiente de hecho el diagnóstico y en el otro al cuarto día. Pericardiectomía se hizo en dos casos a los 17 y 29 días, respectivamente, de hecho el diagnóstico ante la comprobación de signos de taponamiento cardíaco y la verificación en el acto quirúrgico de engrosamiento y adherencias serofibrinosas de las hojas del pericardio. Otro paciente en que se diagnosticó taponamiento cardíaco falleció antes de ser intervenido.

La evolución de los 11 pacientes la consignamos en base a la duración de la hospitalización, mortalidad y secuelas al alta (Tabla 12). La duración de la hospitalización fue de menos de 50 días en seis casos $(54,5 \%)$; de 50 a 100 días en $4(36,5 \%)$ y de más de 100 días en uno $(8 \%)$. Con un promedio total de 46 días.

La mortalidad fue de un $27,3 \%$ (3 casos). Uno de ellos falleció a las pocas horas de ingreso por un grave cuadro séptico y el diagnóstico de pericarditis fue un hallazgo de necropsia. En otro el deceso ocurrío a los dos días de su ingreso por taponamiento cardíaco antes de ser intervenido. El tercero falleció a los 9 días de hospitalización también con un cuadro séptico severo con compromiso pleuropulmonar bilateral.

Ocho niños egresaron en buenas condiciones y en ninguno de ellos se constató pericarditis constrictiva al alta.
TABLA 11

Tratamiento efectuado en 11 pacientes con pericarditis purulenta

\begin{tabular}{lcccc} 
A) Antibióticos usados & Pacientes & \multicolumn{3}{c}{ Duración Días } \\
\hline Cloxacilina & 10 & 38 & y & 104 \\
Meticilina & 2 & 12 & y & 48 \\
Lincomicina & 1 & & & 70 \\
Cefaloridina & 1 & & 60 \\
Cloramfenicol & 5 & 3 & - & 40 \\
Penicilina & 5 & 6 & - & $\mathbf{1 4}$ \\
\hline
\end{tabular}

NotA: gentralmente no se usaron de acuerdo al antibiograma.

B) Pericardiocentesis (en 2 pacientes)

\begin{tabular}{rr}
\hline $1^{\circ}$ & 5 punciones \\
$2^{\circ}$ & 1 punción 2 fueron sometidos a \\
\hline
\end{tabular}

C) Tratamiento quirúrgico. Días de hecho el diagóstico

\begin{tabular}{|c|c|c|}
\hline \multirow{3}{*}{ Pericandiostomía } & Caso $1^{\circ}$ & 4 \\
\hline & $2^{\circ}$ & 1 \\
\hline & $1^{\circ}$ & 17 \\
\hline Pericardiectomía & $2^{\circ}$ & 29 \\
\hline
\end{tabular}

TABLA 12

Evolución en 11 pacientes con pericarditis purulenta

A) Duración de la hospitalización

\begin{tabular}{|c|c|c|c|}
\hline Dias & No casos & $\%$ & OBS. \\
\hline-50 & 6 & 54,5 & \\
\hline $50-100$ & 4 & 36,5 & \\
\hline+100 & 1 & 9,0 & \\
\hline TOTAL & 11 & 100 & Promedio 46 dias \\
\hline
\end{tabular}

B) Mortalidad

\begin{tabular}{lccc}
\hline Alta & No $\operatorname{casos}$ & $\%$ & OBS. \\
\hline Fallecidos & 3 & 27,3 & Dos niños fallecieron \\
Mejorados & 8 & 72,7 & antes de las $48 \mathrm{hrs}$ \\
\hline TOTAL & 11 & 100 & \\
\hline
\end{tabular}

C) Secuelas al alta en 8 pacientes

Pericarditis constictiva 0

Arritmia extrasistólica 1 


\section{COMENTARIO:}

Entre los años 1965 - 1974 ingresaron 8.707 niños a la Unidad de Infecciosos del Servicio de Pediatría del Hospital Guillermo Grant B. de los cuales 11 correspondieron a pericarditis purulenta $(0,10 \%)$. Otros autores como Benzig entre los años 1952-1962 presenta 8 niños con pericarditis purulenta. Horan, entre 1938 y 1956 relata 15 casos de pericarditis estafilocócica. En la literatura nacional son escasas las publicaciones del te$\mathrm{ma}$, lo que dificulta evaluar la verdadera incidencia de esta afección en nuestro medio. En adultos, Donoso comunica entre los años 1946-1962 seis pacientes con pericarditis purulenta. En niños, Pino y cols. relatan 4 pacientes con esta afección en el Hospital R. del Río (1967).

En nuestra serie, esta afección fue un hecho más de un cuadro séptico grave. Habitualmente con varias localizaciones.

La puerta de entrada de la sepsis se pesquisó en seis casos, siendo en su mayoría afecciones cutáneas piógenas. Esto reafirma la necesidad de una conducta vigilante y agresiva frente a estos cuadros.

Se constató una asociación extraordinariamente frecuente con osteomielitis y pleuroneumopatías lo que debe motivarnos a buscar sistemáticamente pericarditis ante la presencia de estos cuadros.

La etiología bacteriana predominante en nuestros casos estuvo constituida por estafilococo aureus lo que concuerda con experiencias nacionales y extranjeras $(1-2-5)$.

En nuestra serie el diagnóstico de pericarditis se realizó dentro de las primeras 48 horas de ingreso en 6 pacientes. Los frotes pericárdicos fueron el signo más preponderante para plantear el diagnóstico clínico.

Queremos destacar la presencia de dolor abdominal previo a la aparición de la signología clínica de pericarditis en 7 pacientes, con un intervalo de 1 a cuatro días. En dos de ellos se pensó en un comienzo en un abdomen agudo. Sobre estos hallazgos coinciden otros autores extranjeros.

El estudio radiológico de tórax sólo demostró aumento de la silueta cardíaca, en muchos casos con forma típica. No se constató en forma relevante otros hechos clásicos como desaparición del ángulo cardiofrénico.

La terapia antibiótica prolongada de acuerdo a la sensibilidad del estudio bacteriológico y el empleo de vaciamiento quirúrgico precoz del pericardio constituyeron, en algunos casos, hechos fundamentales en el tratamiento de nuestros pacientes. La pericardiocentesis no constituyó una medida útil en el tratamiento y en ambos casos en que se efectuó de todas maneras se llegó al vaciamiento quirúrgico.

La duración de la hospitalización fue prolongada, dependiendo más bien de la gravedad de las afecciones concomitantes que del cuadro septicopiohémico.

Ocho de nuestros pacientes evolucionaron en forma satisfactoria egresando sin secuelas.

\section{RESUMEN}

Se presentan 11 casos de Pericarditis purulenta entre los años $1965-1974$. Se destaca la etiología estafilocócica como la más frecuente siendo la pericarditis un hecho más de un cuadro séptico grave.

Se enfatiza que el uso de pericardiostomía y antibioterapia prolongada son fundamentales para lograr éxito en el tratamiento.

No encontramos en nuestros casos Pericarditis constrictiva como secuela al alta.

\section{R E F E R E N C I A S}

1.-Benzig, G.; Kaplan, S.: "Purulent Pericarditis". Am. Jour. Dis. of Children, 106: 289 -294, 1963.

2.-Horan, J. M.: "Acute Staphilococal Pericarditis". Pediatrics, 19: 36 - 41, 1957.

3.-Watson, H.: "Cardiología Pediátrica”, Ed. Salvat, 1970.

4.- Meneghello, Julio. Texto de Pediatría. Intermédica. 1972.

5.-Donoso, P., Donoso, C.: "Tratamiento de la Pericarditis Purulenta". Rev. Med. de Chile, 92: 518520, 1964.

6.-Brent O'Conell, M.; Harrisburg, P.: "Pericarditis following Meningococcia Meningitis". Am. Jour. Dis. of Children. Vol. 126, 265-267, 1973.

7.-Gersony, W. M.; Mc Cracken, G.: "Purulent Pericarditis in infancy”. Pediatrics, 40: 224, 1967.

8.-Rubinstein, J.; Goldlatt, A.: "Acute constriction complicating purulent pericarditis in infancy". Amer. Jour. Dis. of Children, 124: 591-594, 1972.

9.- Pieroni, D.; Panks, Md., Hallbrook, Md.: "Echocardiographic diagnosis of septic pericarditis in infancy". Journal of Pediatrics 82, 689-691, 1973.

10.- Boles and Hossier, Dm.: "Abdominal pain in acute myocarditis and pericarditis". Am. Jour. Dis. of Children, 165: 70-76, 1963.

11.- Fiegenbaum, H.: "Echocardiographic diagnosis of pericardial effusion". Amer. J. Cardiology, 26: 475, 1970.

12.- Feigenbaum, H., Waldhauser, T. A. and Hyde, L.: "Ultrasound diagnosis of pericardial effusion". J.A.M.A., 191: 711, 1965. 